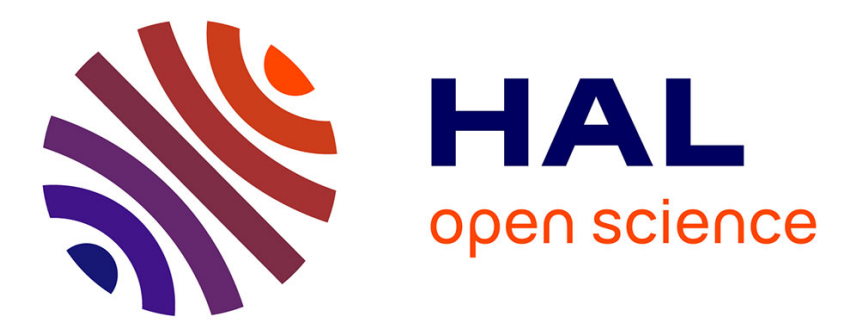

\title{
Radio disappearance of the magnetar XTE J1810-197 and continued X-ray timing
}

\author{
F. Camilo, S. M. Ransom, J. P. Halpern, J. A. J. Alford, Ismaël Cognard, J. \\ E. Reynolds, S. Johnston, J. Sarkissian, W. van Straten
}

\section{- To cite this version:}

F. Camilo, S. M. Ransom, J. P. Halpern, J. A. J. Alford, Ismaël Cognard, et al.. Radio disappearance of the magnetar XTE J1810-197 and continued X-ray timing. The Astrophysical Journal, 2016, 820 (2), pp.110. 10.3847/0004-637X/820/2/110 . insu-01327169

\section{HAL Id: insu-01327169 \\ https://hal-insu.archives-ouvertes.fr/insu-01327169}

Submitted on 6 Apr 2017

HAL is a multi-disciplinary open access archive for the deposit and dissemination of scientific research documents, whether they are published or not. The documents may come from teaching and research institutions in France or abroad, or from public or private research centers.
L'archive ouverte pluridisciplinaire HAL, est destinée au dépôt et à la diffusion de documents scientifiques de niveau recherche, publiés ou non, émanant des établissements d'enseignement et de recherche français ou étrangers, des laboratoires publics ou privés. 


\title{
RADIO DISAPPEARANCE OF THE MAGNETAR XTE J1810-197 AND CONTINUED X-RAY TIMING
}

\author{
F. CAmilo ${ }^{1,2}$, S. M. Ransom ${ }^{3}$, J. P. Halpern ${ }^{1}$, J. A. J. Alford ${ }^{1}$, I. Cognard ${ }^{4,5}$, J. E. Reynolds ${ }^{6}$, S. Johnston ${ }^{6}$, \\ J. SarkisSian ${ }^{7}$, AND W. van STRATEN ${ }^{8}$ \\ ${ }^{1}$ Columbia Astrophysics Laboratory, Columbia University, New York, NY 10027, USA \\ ${ }^{2}$ SKA South Africa, Pinelands, 7405 , South Africa \\ ${ }^{3}$ National Radio Astronomy Observatory, Charlottesville, VA 22903, USA \\ ${ }^{4}$ Laboratoire de Physique et Chimie de l'Environnement et de l'Espace, LPC2E CNRS-Université d'Orléans, F-45071 Orléans, France \\ ${ }^{5}$ Station de radioastronomie de Nançay, Observatoire de Paris, CNRS/INSU F-18330 Nançay, France \\ ${ }^{6}$ CSIRO Astronomy and Space Science, Australia Telescope National Facility, Epping, NSW 1710, Australia \\ ${ }^{7}$ CSIRO Parkes Observatory, Parkes, NSW 2870, Australia \\ ${ }^{8}$ Swinburne University of Technology, Hawthorn, VIC 3122, Australia \\ Received 2015 May 26; accepted 2016 February 16; published 2016 March 28
}

\begin{abstract}
We report on timing, flux density, and polarimetric observations of the transient magnetar and $5.54 \mathrm{~s}$ radio pulsar XTE J1810-197 using the Green Bank, Nançay, and Parkes radio telescopes beginning in early 2006, until its sudden disappearance as a radio source in late 2008. Repeated observations through 2016 have not detected radio pulsations again. The torque on the neutron star, as inferred from its rotation frequency derivative $\dot{\nu}$, decreased in an unsteady manner by a factor of three in the first year of radio monitoring, until approximately mid-2007. By contrast, during its final year as a detectable radio source, the torque decreased steadily by only $9 \%$. The periodaveraged flux density, after decreasing by a factor of 20 during the first 10 months of radio monitoring, remained relatively steady in the next 22 months, at an average of $0.7 \pm 0.3 \mathrm{mJy}$ at $1.4 \mathrm{GHz}$, while still showing day-to-day fluctuations by factors of a few. There is evidence that during this last phase of radio activity the magnetar had a steep radio spectrum, in contrast to earlier flat-spectrum behavior. No secular decrease presaged its radio demise. During this time, the pulse profile continued to display large variations; polarimetry, including of a new profile component, indicates that the magnetic geometry remained consistent with that of earlier times. We supplement these results with X-ray timing of the pulsar from its outburst in 2003 up to 2014. For the first 4 years, XTE J1810 -197 experienced non-monotonic excursions in frequency derivative by at least a factor of eight. But since 2007, its $\dot{\nu}$ has remained relatively stable near its minimum observed value. The only apparent event in the X-ray record that is possibly contemporaneous with the radio shutdown is a decrease of $\approx 20 \%$ in the hot-spot flux in 2008-2009, to a stable, minimum value. However, the permanence of the high-amplitude, thermal X-ray pulse, even after the (unexplained) radio demise, implies continuing magnetar activity.
\end{abstract}

Key words: pulsars: individual (XTE J1810-197, PSR J1809-1943) - stars: neutron

\section{INTRODUCTION}

Magnetars are ultra-highly magnetized neutron stars (inferred surface dipolar field strengths $B_{s} \approx 10^{14-15} \mathrm{G}$ ) that display hugely variable and sometimes very bright $\mathrm{X}$-ray emission powered by their decaying fields (Duncan \& Thompson 1992). This process is reflected in their extremely unsteady rotation. Their magnetic fields cause magnetars to spin down very rapidly, and all those known have long periods, $2<P<12$ s.

Among the 23 known magnetars (Olausen \& Kaspi 2014), ${ }^{9}$ four are known to be transient emitters of radio pulsations. The first to be so identified was the $P=5.54 \mathrm{~s}$ anomalous $\mathrm{X}$-ray pulsar (AXP) XTE J1810-197, discovered in early 2003 following an X-ray outburst (Ibrahim et al. 2004). It is unclear when radio emission started, but pulsations were not present in 1998 and a point source was visible by early 2004 (Halpern et al. 2005). Radio pulsations were detected in early 2006 (Camilo et al. 2006), with some properties that are markedly different from those of ordinary rotation-powered pulsars, including extremely variable flux densities and pulse profiles, and flat spectra (e.g., Camilo et al. 2007c; Lazaridis et al. 2008). The emission is also highly linearly polarized, like that of several ordinary young radio pulsars (Camilo et al. 2007d;

\footnotetext{
9 http://www.physics.mcgill.ca/ pulsar/magnetar/main.html
}

Kramer et al. 2007). To a great extent, these properties are shared by all four radio magnetars identified so far (Camilo et al. 2007b, 2008; Levin et al. 2010; Keith et al. 2011; Shannon \& Johnston 2013).

The radio emission from XTE J1810-197 arose following the X-ray outburst. The X-ray flux then decayed exponentially and returned to pre-outburst levels in 2007-2008 (Bernardini et al. 2011). Here we show that XTE J1810-197 ceased to emit detectable radio pulsations in late 2008, and present the timing, flux density, polarimetric, and pulse profile behavior during its last 20 months of radio activity. We also present X-ray timing measurements and fluxes through 2014.

\section{RADIO OBSERVATIONS}

Our previously published radio studies of XTE J1810-197 (also known as PSR J1809-1943) are based on extensive data sets largely from 2006. Here we present results based on observations done with the Robert C. Byrd Green Bank Telescope (GBT), the Nançay radio telescope (NRT), and the CSIRO Parkes telescope, mainly through the end of 2008. Table 1 summarizes relevant parameters for all the radio observations presented in this paper. 
Table 1

Parameters and Sensitivities for Radio Observations of XTE J1810-197

\begin{tabular}{lcccc}
\hline \hline & NRT & PKS AFB & PKS DFB & GBT \\
\hline Center frequency $(\mathrm{MHz})$ & 1398 & 1374 & 1369 & 1950 \\
Bandwidth $(\mathrm{MHz})$ & 64 & 288 & 256 & 600 \\
Gain, $G\left(\mathrm{~K} \mathrm{Jy}^{-1}\right)$ & $1.55^{\mathrm{a}}$ & $0.735^{\mathrm{b}}$ & $0.735^{\mathrm{b}}$ & $1.9^{\mathrm{c}}$ \\
System temperature, $T_{\text {sys }}(\mathrm{K})$ & $47^{\mathrm{d}}$ & 44 & 44 & 28 \\
SEFD $^{\mathrm{e}}(\mathrm{Jy})$ & $30^{\mathrm{f}}$ & $60^{\mathrm{g}}$ & $60^{\mathrm{g}}$ & $15^{\mathrm{h}}$ \\
$\eta$ & 1.0 & $\sqrt{(\pi / 2)^{\mathrm{i}}}$ & 1.0 & $1.2^{\mathrm{j}}$ \\
SEFD $_{\text {eff }}^{\mathrm{k}}(\mathrm{Jy})$ & 30 & 76 & 60 & 17 \\
\hline
\end{tabular}

Notes. Observations used the Berkeley-Orléans-Nançay (BON) coherent dedispersor (Cognard \& Theureau 2006) at the NRT, the analog (AFB; Manchester et al. 2001) and digital (DFB; Manchester et al. 2013) filterbanks at Parkes, and the pulsar Spigot (Kaplan et al. 2005) at the GBT. Sensitivity parameters are given in the direction of XTE J1810-197 for the specified frequencies and bandwidths. The sky temperature at $1.4 \mathrm{GHz}$ in this direction is $T_{\text {sky }}=16.4 \mathrm{~K}$ including CMB (obtained from http://www3.mpifr-bonn.mpg. de/survey.html; Reich et al. 2001).

a Theureau et al. (2005).

b Nominal $G$ of multibeam receiver center pixel (Manchester et al. 2001).

${ }^{c}$ http://www.gb.nrao.edu/ fghigo/gbtdoc/sens.html.

d $T_{\text {sky }}$ plus "no-sky" $T_{\text {sys }}=30 \mathrm{~K}$ (given cold-sky $T_{\text {sys }}=35 \mathrm{~K}$; http: $/ /$ www. nrt.obspm.fr/nrt/obs/NRT_tech_info.html).

e System equivalent flux density ( $\left.\equiv T_{\text {sys }} / G\right)$.

${ }^{\mathrm{f}}$ Computed from $T_{\text {sys }}$ and known $G$.

g Calibrated assuming Hydra A flux density of $42.5 \mathrm{Jy}$ at $1.4 \mathrm{GHz}$ (after accounting for a $1.5 \%$ beam dilution factor; Baars et al. 1977).

${ }^{\mathrm{h}}$ Measured within the Spigot band from flux-calibrated GUPPI observation (https://safe.nrao.edu/wiki/bin/view/CICADA/GUPPiUsersGuide).

${ }^{i}$ Inefficiency factor due to AFB 1-bit sampling (Manchester et al. 2001).

${ }^{\mathrm{j}}$ Estimated inefficiency factor due to Spigot 3-level quantization (cf. Kaplan et al. 2005).

${ }^{\mathrm{k}}$ Effective SEFD ( $\left.\equiv \eta \times \mathrm{SEFD}\right)$, used to compute XTE J1810-197 flux densities (see Sections 2.2 and 2.3).

\subsection{Radio Timing}

In Camilo et al. (2007a) we showed the timing behavior of XTE J1810-197 through 2007 January, based largely on 1.4 GHz data collected with the BON spectrometer at the NRT. As the flux density decreased it became preferable to time the pulsar at the GBT. We did this at $2 \mathrm{GHz}$ using the Spigot spectrometer, recording the data in search mode and folding offline. Nevertheless, BON timing continued to be important, particularly during 2007 May-August when the GBT was not available. The NRT observations were coherently dedispersed, with the full band divided into 16 frequency channels, then folded at the predicted pulsar period with 2-minute subintegrations before mid-2007 and $30 \mathrm{~s}$ thereafter. See Table 2 for a $\log$ of the timing observations newly presented here. Daily observations typically lasted $1 \mathrm{hr}$ at Nançay and varied greatly at the GBT, from 0.25 to $2 \mathrm{hr}$ with most at least $0.5 \mathrm{hr}$ (i.e., from a couple hundred to more than 1000 pulsar rotations per session).

In principle radio timing of XTE J1810-197 involves unusual challenges because of changing pulse profiles. In practice we found that on the vast majority of days a simple procedure was sufficient to obtain times-of-arrival (TOAs) that could be used to reliably describe the rotation of the star. We first excised strong radio frequency interference (RFI) from the BON and Spigot data. BON timing was detailed in Camilo et al. (2007a). For Spigot we obtained TOAs by crosscorrelating individual folded pulse profiles with a Gaussian

\footnotetext{
${ }^{10}$ http://tempo.sourceforge.net
}

Table 2

Log of Radio Timing Observations of XTE J1810-197

\begin{tabular}{lcc}
\hline \hline MJD Range (days) & Number of Daily TOAs & Telescope \\
\hline $54128-54218(90)$ & $53+18$ & GBT + Nançay \\
$54226-54357(131)$ & 27 & Nançay \\
$54352-54739(387)$ & 51 & GBT \\
\hline
\end{tabular}

Note. GBT TOAs were obtained with Spigot at $2 \mathrm{GHz}$; Nançay TOAs were obtained with BON at $1.4 \mathrm{GHz}$ (see Section 2.1). The average observing cadence in the three periods listed decreased from once every 1.5 days to once every 5 days to once every 7.5 days.
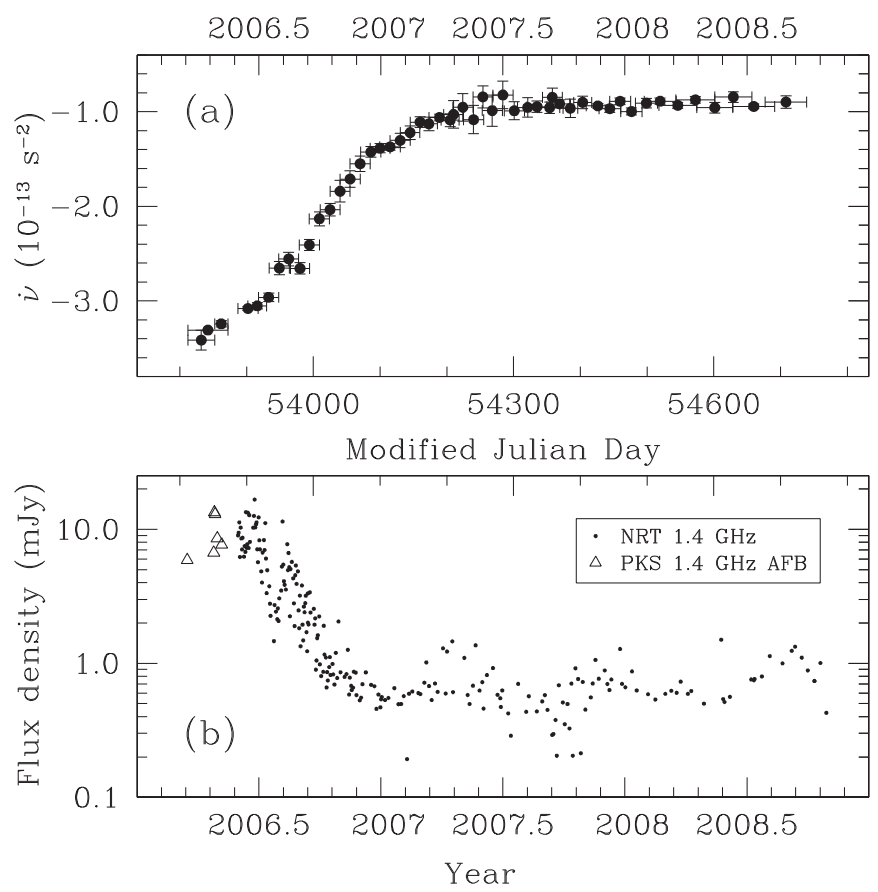

Figure 1. Torque and radio flux density of XTE J1810-197. (a) Frequency derivative vs. date (Section 2.1). Measurements from before MJD 54127 (2007.1) are reproduced from Camilo et al. (2007a). (b) Period-averaged flux density vs. date. Data from before 2007.1 were presented in Camilo et al. (2007a) but have been reanalyzed for this paper (see Section 2.2).

template. We then used the TOAs with the TEMPO software ${ }^{10}$ to obtain timing solutions.

The position of XTE J1810-197 was held fixed in all our timing fits at that determined from very long baseline interferometry (VLBI) observations (Helfand et al. 2007); the measured proper motion is too small to affect the timing of this pulsar. As explained in Camilo et al. (2007a), we maintained phase connection for this pulsar since 2006 April, but the rotation frequency derivative was changing so rapidly that it proved more informative to measure $\dot{\nu}$ (where $\nu=1 / P$ ) using TOAs typically spanning one month, and doing a TEMPO fit for only $\nu$ and $\dot{\nu}$. We did such fits in segments of data offset by roughly 15 days to provide a good sampling of $\dot{\nu}$, and the results are shown in Figure 1(a). In this panel, the first 17 measurements are reproduced from Camilo et al. (2007a), while the next 33 measurements are new.

It is clear from Figure 1(a) that over the last $\sim 400$ days $\dot{\nu}$ stabilized greatly, compared with earlier large variations. To investigate this in detail we obtained phase-connected fits spanning the last year of timing data. In Figure 2(a) we show the (large) residuals from a simple fit to rotation phase, $\nu$, and $\dot{\nu}$. 


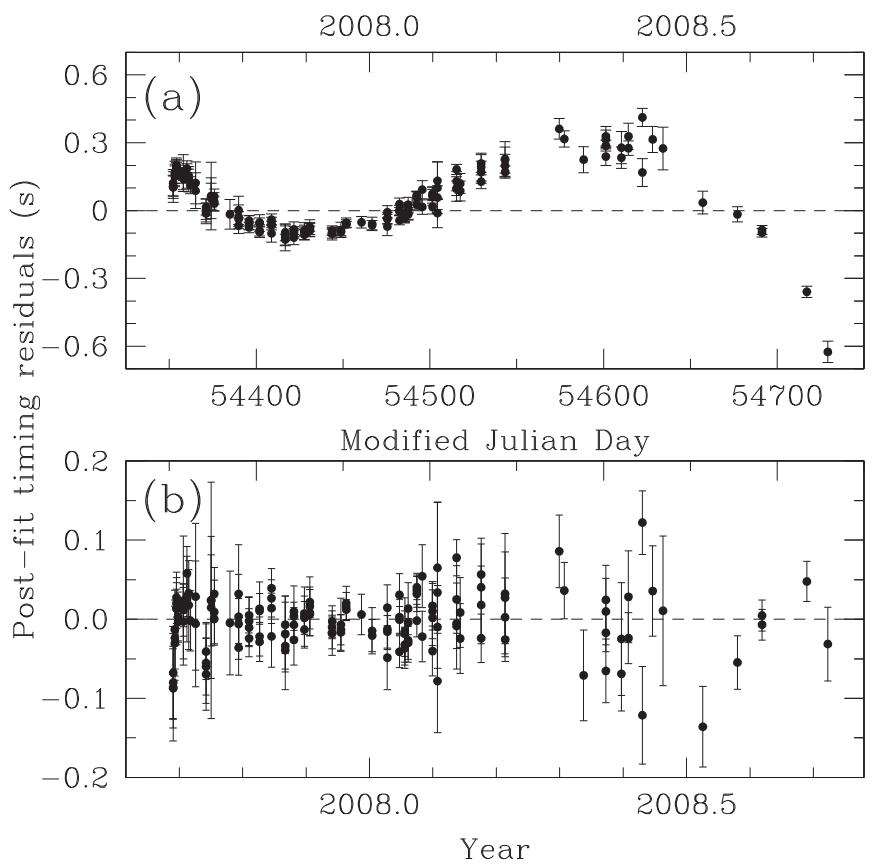

Figure 2. Timing residuals for XTE J1810-197. (a) Phase residuals vs. date for a timing model that fits only for rotation phase, frequency, and frequency derivative, showing a cubic trend. (b) Residuals for a model that fits for phase, $\nu, \dot{\nu}$, and $\ddot{\nu}$ (see Section 2.1 and Table 3).

In Figure 2(b), we see that the addition of $\ddot{\nu}$ absorbs all remaining residual trends. These timing solutions are listed in Table 3 . The positive value of $\ddot{\nu}$ implies that during this span $-\dot{\nu}$ (proportional to the braking torque) was decreasing steadily, by a total of $9 \%$ during the year.

\subsection{Radio Flux Densities}

One unusual aspect of radio emission from XTE J1810-197 is the fluctuation on $\sim$ daily timescales of its period-averaged flux density, which is largely intrinsic to the pulsar (e.g., Lazaridis et al. 2008). These variations result from a combination of different pulse profile components becoming active (i.e., because of radically changing profiles) and varying intensity from particular components. Superimposed on this apparently chaotic variation, the average flux density of the pulsar decreased by more than an order of magnitude in the 10 months following its pulsed radio discovery, as seen in Figure 1(b) (these pre-2007 data were originally presented in Camilo et al. 2007a, but have been reanalyzed here to present a consistent flux density record).

Most period-averaged flux densities presented in this paper (including all those in Figure 1(b)) were obtained by measuring, for each daily observation, the area under the pulse profile, scaled to its off-pulse rms, and converting to a Jansky scale using the observing parameters and telescope system

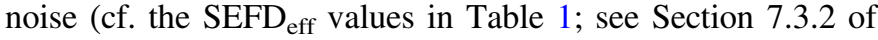
Lorimer \& Kramer 2004 for more details on this method). We estimate that the absolute $1.4 \mathrm{GHz}$ flux density scale is accurate to within $10 \%$.

The greatest source of uncertainty arises from the impact of RFI and system noise fluctuations on this long period and, from 2007, faint pulsar. Each pulse profile was carefully excised of RFI in both the frequency and time domains. Nevertheless, for approximately a third of all the post-2006 NRT observations,
Table 3

Two Radio Timing Solutions for XTE J1810-197

\begin{tabular}{ll}
\hline \hline Parameter & Value \\
\hline R.A. (J2000.0) & $18^{\mathrm{h}} 09^{\mathrm{m}} 51^{\mathrm{s}} .087$ \\
Decl. (J2000.0) & $-19^{\circ} 43^{\prime} 51^{\prime \prime} \cdot 93$ \\
Dispersion measure, DM & $178.0 \mathrm{pc} \mathrm{cm}^{-3}$ \\
Epoch (MJD TDB) & 54550.0 \\
Range of dates (MJD) & $54352-54729$ \\
Frequency, $\nu^{\mathrm{a}}$ & $0.18048830377(8) \mathrm{Hz}$ \\
Frequency derivative, $\dot{\nu}^{\mathrm{a}}$ & $-9.163(1) \times 10^{-14} \mathrm{~Hz} \mathrm{~s}^{-1}$ \\
\hline Frequency, $\nu^{\mathrm{b}}$ & $0.1804882977(1) \mathrm{Hz}^{-14} \mathrm{~Hz} \mathrm{~s}^{-1}$ \\
Frequency derivative, $\dot{\nu}^{\mathrm{b}}$ & $-9.090(2) \times 10^{-14} \mathrm{~Hz} \mathrm{~s}^{-2}$ \\
Frequency second derivative, $\ddot{\nu}^{\mathrm{b}}$ & $2.46(5) \times 10^{-22} \mathrm{~Hz}^{\mathrm{b}}$ \\
rms post-fit timing residual $(P)$ & 0.007 \\
\hline
\end{tabular}

Notes. The celestial coordinates were held fixed at the values obtained from VLBA observations (Helfand et al. 2007), and the DM was held fixed at the value obtained from simultaneous 0.7 and $2.9 \mathrm{GHz}$ observations (Camilo et al. 2006).

a These two parameters are sufficient to obtain a phase-connected solution encompassing the MJD range, but do not fully describe the rotation of the neutron star. See Figure 2(a) and Section 2.1.

$\mathrm{b}$ These three parameters fully describe the rotation of the neutron star within the given MJD range, but have little predictive value outside it. See Figure 2(b) and Section 2.1 .

the RFI was so bad or the flux density was so low (approaching the $\approx 0.1 \mathrm{mJy}$ detection threshold), that we did not extract a flux density measurement at all. In some of the remaining instances, as much as half of the data had to be discarded in order to obtain an integrated profile clean enough to measure flux density. Mindful of these caveats (most often some residual RFI is bound to remain, and individual measurements could be greatly affected), we estimate that the NRT flux density measurements in Figure 1(b) have a typical relative fractional uncertainty of $\approx 20 \%$, with a minimum of $0.1 \mathrm{mJy}$.

Two things immediately stand out from Figure 1(b): daily flux density variations continued through the end of our data set by factors of a few, and the average $1.4 \mathrm{GHz}$ flux density stabilized early in 2007 at a level of $0.68 \pm 0.27 \mathrm{mJy}$ for all post-2006 NRT detections presented here. In addition, the panels of Figure 1 are something of a mirror image of each other.

\subsubsection{Radio Disappearance}

With no warning from either its timing or flux density behavior, XTE J1810-197 ceased to emit detectable radio pulsations in late 2008. The last detection at the NRT was on October 29, on November 2 at the GBT (Figure 3), and at Parkes on the following day. The next attempt to detect it was on November 10. We attempted to detect the pulsar at Parkes on 20 occasions through 2016 January, largely at $1.4 \mathrm{GHz}$, each time for $0.5-1 \mathrm{hr}$. At the NRT we did 10 more observations through 2009 June. At the GBT we made a total of 17 attempts at $2 \mathrm{GHz}$, each $\approx 0.5 \mathrm{hr}$, through 2012 August. The pulsar was not detected in any of the 47 observations spanning 7 years since 2008 November (Figure 4). We emphasize that XTE J1810-197 did not gradually fade into undetectability. A few weeks before the last detection, we recorded beautiful profiles (e.g., Figures 6(d), 7(c) and (d)); the signal strength and pulse profiles were fluctuating at least as 

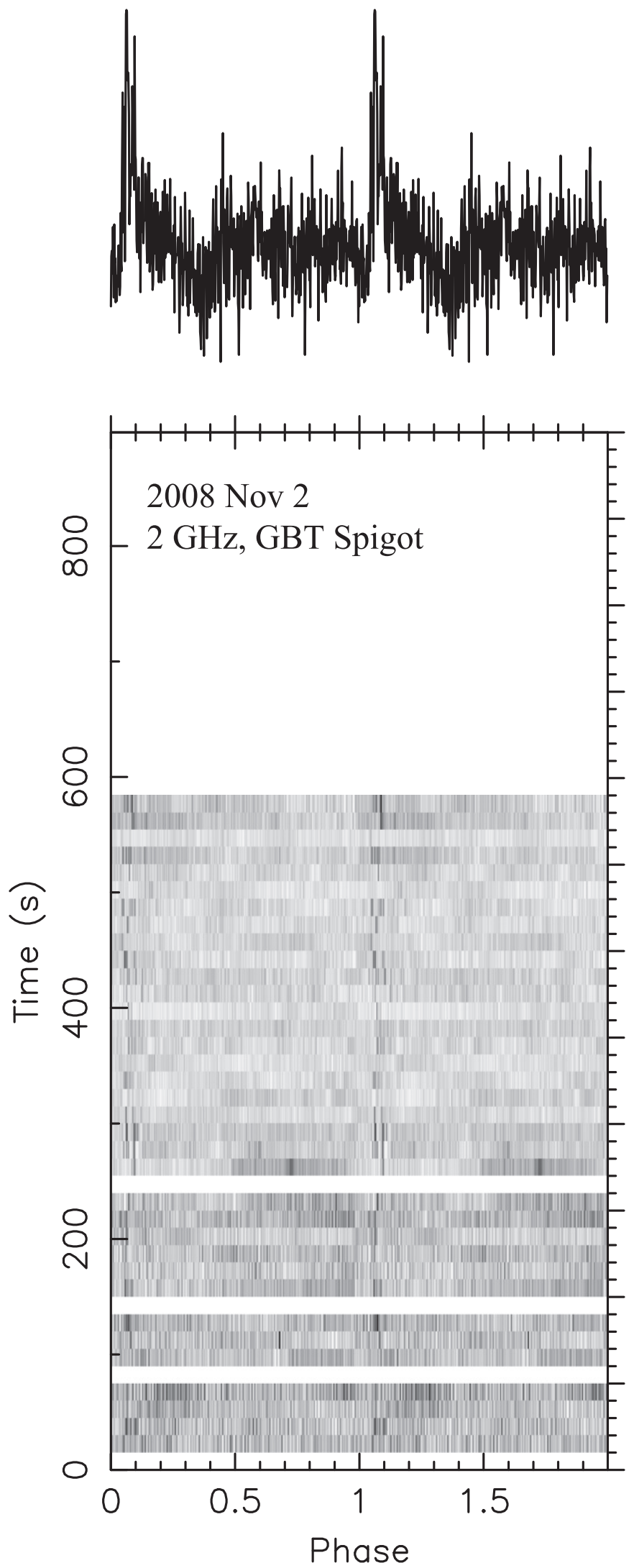

Figure 3. Last radio detection of XTE J1810-197 at the GBT. The periodaveraged flux density is among the smallest we observed $(\sim 50 \mu \mathrm{Jy})$, but pulse detection is aided by the "spiky" nature of its narrow subpulses. Two rotations are shown as a function of time, with the summed profile at the top. White areas are subintegrations masked due to particularly bad RFI.

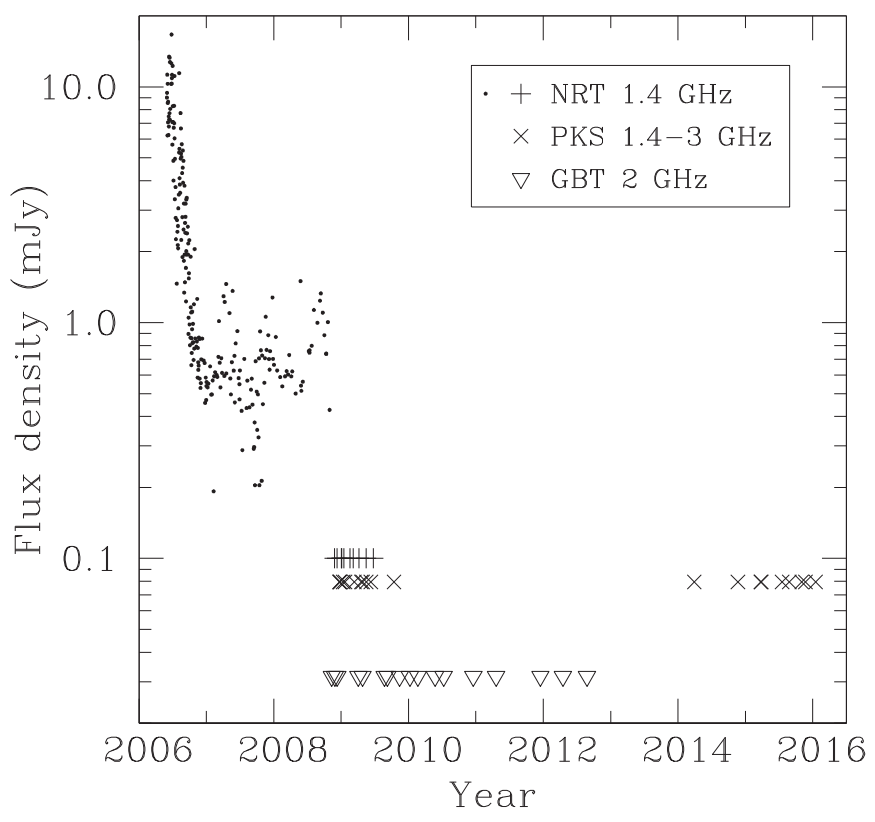

Figure 4. Shown are 47 radio nondetections of XTE J1810-197 since late 2008 (Section 2.2.1). The dots represent measured NRT flux densities, reproduced from Figure 1(b). The other symbols denote individual observations without a detection, placed at approximately the upper flux density limits for the respective sets of observations.

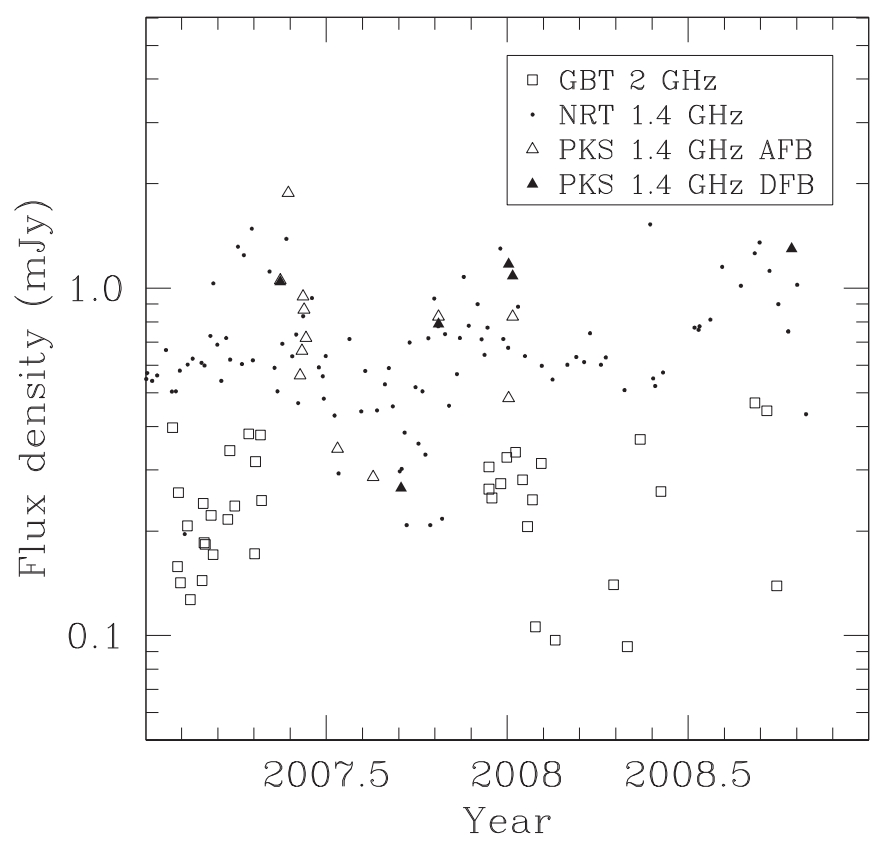

Figure 5. Flux density measurements for XTE J1810-197 at 1.4 and $2 \mathrm{GHz}$ in 2007-2008. Parkes observations include those done with analog (AFB) and full-Stokes digital (DFB) filterbanks. See Section 2.3 for details.

much as they had for the previous $\sim 500$ days. And then the radio pulses were gone.

For an assumed pulse duty cycle of $6 \%$ (comparable to the component widths often observed for this pulsar) and the parameters used in our monitoring observations (Table 1), the upper limit on the flux density of XTE J1810-197 since late 2008 is approximately $0.1 \mathrm{mJy}$ at $1.4 \mathrm{GHz}$ based on the NRT observations, slightly lower than that for the Parkes 
observations, and approximately $0.03 \mathrm{mJy}$ at $2 \mathrm{GHz}$ based on the GBT observations (Figure 4). These limits are nearly an order of magnitude below the average flux densities during the 1.8 years of radio emission following 2006, although they are not much below the faintest established detections (see Figures 3 and 5). The great profile variability, long period, and RFI make more detailed estimates unreliable. In any case, in none of 47 observations spanning 7 years since the last detection in late 2008, was the pulsar as detectable as in the poorest of more than 200 detections made over the 2-year period before 2008 November.

\subsection{Radio Spectrum}

The available evidence suggests that during the faint epoch that lasted for 2 years preceding its radio disappearance in late 2008, XTE J1810-197 had a steep radio spectrum, contrasting to its earlier generally flat spectrum.

In Figure 5 we present our post-2006 flux density measurements at 1.4 and $2 \mathrm{GHz}$. The $1.4 \mathrm{GHz}$ NRT values are reproduced from Figure 1(b). These are fundamentally consistent with Parkes measurements at the same frequency, which were obtained from six full-Stokes observations using pulsar digital filterbanks (DFBs) analyzed with PSRCHIVE (Hotan et al. 2004) and 12 analog filterbank (AFB) observations.

The $2 \mathrm{GHz}$ flux density values presented here were obtained from a subset of the GBT data used to derive TOAs (Section 2.1), using the method outlined in Section 2.2 for NRT and Parkes AFB observations. We determined the system noise (Table 1) from a full-Stokes $2 \mathrm{GHz}$ observation in the direction of XTE J1810-197, flux-calibrated with PSRCHIVE. Even after careful RFI excision, we selected for reliable flux density measurements only $40 \%$ of all observations from which we extracted a TOA. We estimate relative fractional uncertainties of $\approx 20 \%$ on average.

The $2 \mathrm{GHz}$ measurements in Figure 5 range over $0.1-0.5 \mathrm{mJy}$, with an average and standard deviation of $S_{2}=0.25 \pm 0.10 \mathrm{mJy}$. This is to be compared to $S_{1.4}=$ $0.68 \pm 0.27 \mathrm{mJy}$ for the $1.4 \mathrm{GHz}$ measurements in the figure (Section 2.2), which span much the same time interval. At face value this would seem to suggest a spectral index of $\alpha \approx-3$ (where $S_{\nu} \propto \nu^{\alpha}$ ). Given the inherent difficulties in extracting such a measurement for this variable pulsar from non-simultaneous multi-frequency observations susceptible to RFI despite our best efforts, we do not claim a reliable numerical value for $\alpha$. However, Figure 5 strongly suggests that XTE J1810-197 became a steep-spectrum object during its final "weak" state prior to its disappearance as a radio source; in that respect it was more akin to an ordinary pulsar than in its earlier "high" state, when the torque was also varying rapidly (Figure 1).

\subsection{Polarimetry}

All of our previously published XTE J1810-197 polarimetric data are from before 2006 December (Camilo et al. 2007d). The data published by Kramer et al. (2007) end even earlier, but include single-pulse polarimetry. Here we present some polarimetric observations from 2007 and 2008 with Parkes at $1.4 \mathrm{GHz}$ and $3 \mathrm{GHz}$, using, respectively, the center beam of the multibeam receiver and the $10 \mathrm{~cm}$ band of the
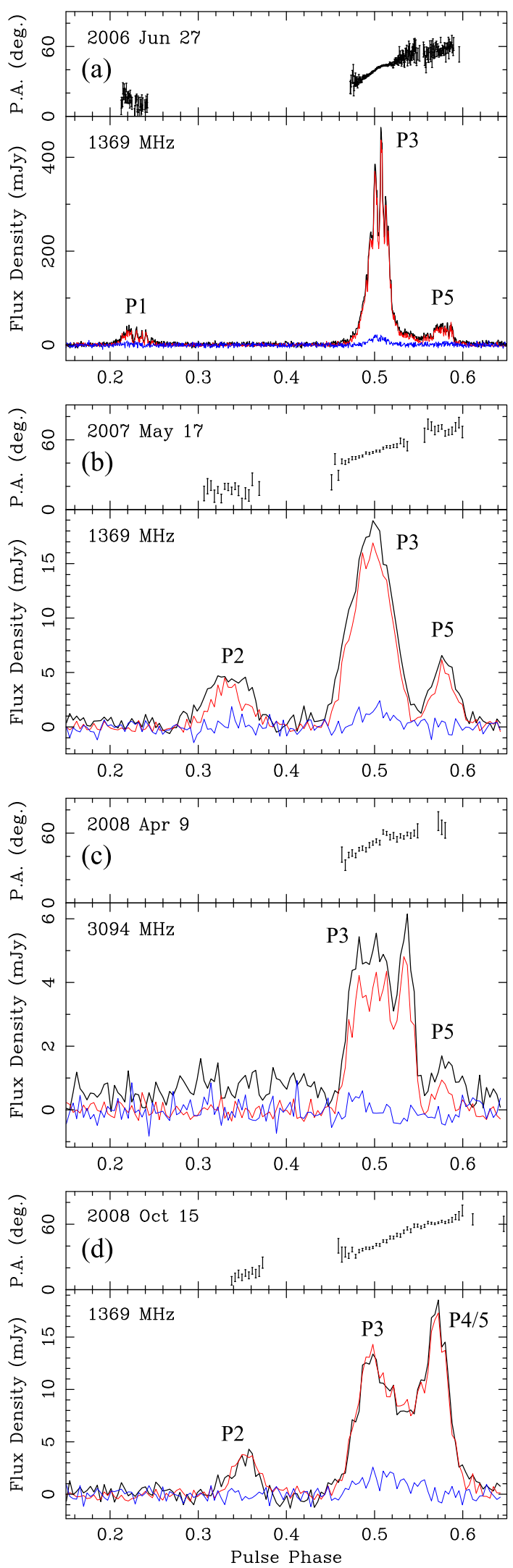

Figure 6. Polarimetric pulse profiles of XTE J1810-197 recorded at Parkes. Only $50 \%$ of the pulse phase is shown. In each lower panel the black trace represents total intensity, red is linear polarization, and blue is circular. Each upper panel shows the position angle of linear polarization, rotated to the pulsar frame using $\mathrm{RM}=78 \mathrm{rad} \mathrm{m}^{-2}$. The profiles are phase-aligned with respect to each other by eye, and absolute phase is arbitrary. Recurring profile features are labeled P1-P5 (as in Figure 7). 
$1050 \mathrm{~cm}$ receiver. The data were collected using DFBs and analyzed with PSRCHIVE, as in Camilo et al. (2007d).

Figure 6(a) is a reprocessed version of a 2006 observation presented in Camilo et al. (2007d), differing mainly in the flux density scale and amount of data excised due to RFI. The recalculated rotation measure is $\mathrm{RM}=76 \pm 1 \mathrm{rad} \mathrm{m}^{-2}$, which is entirely consistent with the value in Camilo et al. (2007d); the RMs calculated for the later observations are consistent with this value within their larger uncertainties.

The $3 \mathrm{GHz}$ full-Stokes profile (Figure 6(c)) looks similar to its counterpart from a year and a half earlier (Figure 1(c) of Camilo et al. 2007d). At $1.4 \mathrm{GHz}$, the two $2007 / 2008$ profiles (Figures 6(b) and (d)) show very similar position angles of linear polarization (PA), and differ mainly in the relative amplitudes of three total intensity profile components (labeled $\mathrm{P} 2$, P3, and $\mathrm{P} 4 / 5$ ). Both profiles are close to $100 \%$ linearly polarized.

Comparison between the 2006 profile (Figure 6(a)) and the $2007 / 20081.4 \mathrm{GHz}$ profiles shows that the PA sweep and its absolute values are similar for the "main pulse" regions (components P3-P5). However, the 2007/2008 profiles show component P2 not present in the 2006 profile-whose $\mathrm{P} 1$ component in turn is not seen later on. Profile components P1-P5 span $40 \%$ of pulse phase, and if they all display stable PAs between 2006 and $2007 / 2008$, then in principle this allows for a more conclusive investigation of geometry than previously possible (see Section 4.1).

\subsection{Radio Pulse Profiles}

The total intensity pulse profiles of XTE J1810-197 were always extremely variable, both in phase of active emitting regions and in the daily appearance of each pulse profile component. The description that follows is based on a review of hundreds of daily profiles that we obtained during 2006-2008 at the GBT, Parkes, and Nançay. There was never any stable pulse profile, but we detected emission from some pulse longitudes more often than from others with some discernible patterns over time, and radio pulsations were never detected from many longitudes. In that sense, there was some long-lasting stability to the radio-emitting region.

Referring to Figure 7(a), emission was always detected from the main pulse region (MP, which in turn was made up of at least three discernible substructures, labeled P3-P5, not all necessarily emitting at once). Emission from component P1 (see also Figure 6(a)) was detected only during 2006, and it was the most variable, sometimes being much brighter than the MP components. On the other hand, P2 was active sometimes in 2006 and 2007 (Figure 6(b)), but became more common in 2008 (Figures 6(d) and 7(c)-(d)). In 2007 the observed profile often consisted of emission from the MP region alone (Figure 7(b)). In only one (2006) observation out of hundreds did we detect emission from all these regions at once (Figure 7(a)).

The relative amplitudes of the different components varied widely; this is exemplified in Figure 7(d) by P4, which was usually not preeminent but was the brightest of any on this day. The very "spiky" nature of the individual subpulses that built up the broader integrated profile components (see, e.g., Serylak et al. 2009) continued to the very end (Figure 3).

Our sense is that in 2007-2008 the profiles were less variable than in 2006, but this impression may be biased by two factors:
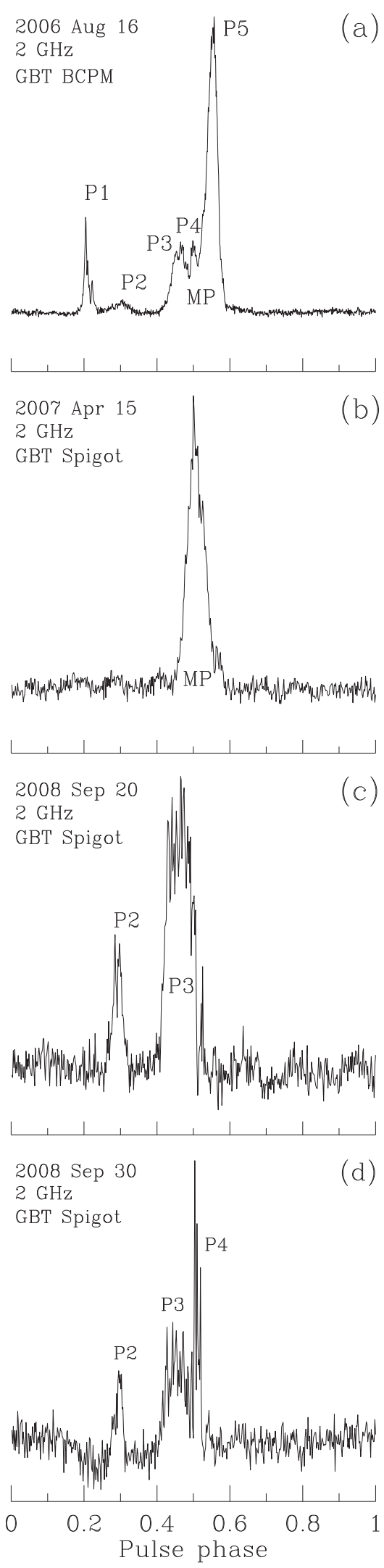

Figure 7. Selection of XTE J1810-197 radio pulse profiles. Prominent features in the profile that reoccur are labeled P1-P5 (as in Figure 6). We define the "main pulse" (MP) region as being composed of up to features P3-P5. Profiles are aligned by eye, and the absolute phase here is arbitrary. 
Table 4

Log of X-Ray Timing Observations of XTE J1810-197

\begin{tabular}{|c|c|c|c|c|c|}
\hline Mission/Instrument & ObsID & $\begin{array}{l}\text { Date } \\
\text { (UT) }\end{array}$ & $\begin{array}{l}\text { Epoch } \\
\text { (MJD) }\end{array}$ & $\begin{array}{l}\text { Exposure } \\
\text { (ks) }\end{array}$ & $\begin{array}{l}\text { Frequency } \\
(\mathrm{Hz})\end{array}$ \\
\hline Chandra HRC & 4454 & 2003 Aug 27 & 52878 & 2.8 & $0.180531(11)$ \\
\hline XMM-Newton pn+MOS & 0161360301 & 2003 Sep 8 & 52890 & 12.1 & $0.18052682(30)$ \\
\hline XMM-Newton pn & 0152833201 & 2003 Oct 12 & 52924 & 8.9 & $0.1805245(12)$ \\
\hline Chandra HRC & 5240 & 2003 Nov 1 & 52944 & 2.8 & $0.180536(10)$ \\
\hline XMM-Newton $\mathrm{pn}+\mathrm{MOS}$ & 0161360501 & 2004 Mar 11 & 53075 & 18.9 & $0.18052415(25)$ \\
\hline XMM-Newton pn+MOS & 0164560601 & 2004 Sep 18 & 53266 & 28.9 & $0.18051856(18)$ \\
\hline XMM-Newton $\mathrm{pn}+\mathrm{MOS}$ & 0301270501 & 2005 Mar 18 & 53447 & 42.2 & $0.18051142(16)$ \\
\hline XMM-Newton $\mathrm{pn}+\mathrm{MOS}$ & 0301270401 & 2005 Sep 20 & 53633 & 42.2 & $0.1805046(3)$ \\
\hline XMM-Newton $\mathrm{pn}+\mathrm{MOS}$ & 0301270301 & 2006 Mar 12 & 53806 & 51.4 & $0.1804991(4)$ \\
\hline Chandra ACIS-S & 6660 & 2006 Sep 10 & 53988 & 30.1 & $0.1804942(14)$ \\
\hline XMM-Newton $\mathrm{pn}+\mathrm{MOS}$ & 0406800601 & 2006 Sep 24 & 54002 & 50.3 & $0.18049355(34)$ \\
\hline XMM-Newton $\mathrm{pn}+\mathrm{MOS}$ & 0406800701 & 2007 Mar 6 & 54165 & 68.3 & $0.18049117(27)$ \\
\hline XMM-Newton $\mathrm{pn}+\mathrm{MOS}$ & 0504650201 & 2007 Sep 16 & 54359 & 74.9 & $0.18048987(19)$ \\
\hline Chandra ACIS-S & 7594 & 2008 Mar 18 & 54543 & 29.6 & $0.1804868(15)$ \\
\hline XMM-Newton $\mathrm{pn}+\mathrm{MOS}$ & 0552800201 & 2009 Mar 5 & 54895 & 65.8 & $0.18048610(24)$ \\
\hline XMM-Newton pn+MOS & 0605990201 & 2009 Sep 5 & 55079 & 21.6 & $0.1804857(13)$ \\
\hline XMM-Newton $\mathrm{pn}+\mathrm{MOS}$ & 0605990301 & 2009 Sep 7 & 55081 & 19.9 & $0.1804829(16)$ \\
\hline XMM-Newton pn+MOS & 0605990401 & 2009 Sep 23 & 55097 & 14.2 & $0.1804872(24)$ \\
\hline Chandra ACIS-S & 11102 & 2009 Nov 1 & 55136 & 25.1 & $0.1804852(16)$ \\
\hline Chandra ACIS-S & 12105 & 2010 Feb 15 & 55242 & 12.6 & $0.180476(6)$ \\
\hline Chandra ACIS-S & 11103 & 2010 Feb 17 & 55244 & 12.6 & $0.180484(6)$ \\
\hline XMM-Newton $\mathrm{pn}+\mathrm{MOS}$ & 0605990501 & 2010 Apr 9 & 55295 & 9.9 & $0.1804820(48)$ \\
\hline Chandra ACIS-S & 12221 & 2010 Jun 07 & 55354 & 10.0 & $0.180489(8)$ \\
\hline XMM-Newton $\mathrm{pn}+\mathrm{MOS}$ & 0605990601 & 2010 Sep 5 & 55444 & 11.3 & $0.1804771(40)$ \\
\hline Chandra ACIS-S & 13149 & 2010 Oct 25 & 55494 & 15.4 & $0.1804766(35)$ \\
\hline Chandra ACIS-S & 13217 & 2011 Feb 8 & 55600 & 15.0 & $0.1804835(35)$ \\
\hline XMM-Newton $\mathrm{pn}+\mathrm{MOS}$ & 0671060101 & 2011 Apr 3 & 55654 & 22.9 & $0.1804796(13)$ \\
\hline XMM-Newton pn+MOS & 0671060201 & 2011 Sep 9 & 55813 & 15.9 & $0.1804751(18)$ \\
\hline Chandra ACIS-S & 13746 & 2012 Feb 19 & 55976 & 20.0 & $0.1804790(23)$ \\
\hline Chandra ACIS-S & 13747 & 2012 May 24 & 56071 & 20.0 & $0.1804774(21)$ \\
\hline XMM-Newton $\mathrm{pn}+\mathrm{MOS}$ & 0691070301 & 2012 Sep 6 & 56176 & 17.9 & $0.1804721(17)$ \\
\hline XMM-Newton $\mathrm{pn}+\mathrm{MOS}$ & 0691070401 & 2013 Mar 3 & 56354 & 17.9 & $0.1804722(18)$ \\
\hline XMM-Newton $\mathrm{pn}+\mathrm{MOS}$ & 0720780201 & 2013 Sep 5 & 56540 & 24.5 & $0.1804721(12)$ \\
\hline ChandraACIS-S & 15870 & 2014 Mar 1 & 56717 & 20.1 & $0.1804723(20)$ \\
\hline XMM-Newton $\mathrm{pn}+\mathrm{MOS}$ & 0720780301 & 2014 Mar 4 & 56720 & 25.0 & $0.1804682(11)$ \\
\hline Chandra ACIS-S & 15871 & 2014 Sep 7 & 56911 & 20.1 & $0.1804710(23)$ \\
\hline
\end{tabular}

the pulsar was much brighter in 2006, which allowed the detection of very faint rarely observed components (see Figure 2 of Camilo et al. 2007a), and the total integration (both in number and in average duration of observations) was larger in 2006 compared with later. In any case, it is clear that during the period when the torque had stabilized by comparison to earlier huge variations, and when the period-averaged flux density had also stabilized in an average sense at a low level (Figure 1), the pulse profiles of XTE J1810-197 were still varying at unprecedented levels compared with normal pulsars, and they continued to do so until radio pulsations disappeared.

\section{X-RAY OBSERVATIONS}

In order to search for clues to the disappearance of radio pulsations from XTE J1810-197, we reviewed all its archival chandra and XMM-Newton data collected from 2003 to 2014. Timing results from 2009 November onward are newly published here. Detailed spectroscopic and flux analysis are presented in Alford \& Halpern (2016), with a summary of the fluxes given in Section 3.2. In summary, we find that the X-ray fluxes stepped down to a minimum value around the time that the radio pulsations shut off, and this is the only recognizable event in X-rays that is plausibly contemporaneous with the disappearance in radio.

\subsection{X-Ray Timing}

Table 4 is a log of all XTE J1810-197 timing observations performed by Chandra and XMM-Newton through 2014. The Chandra ACIS observations were taken with the source on the S3 CCD, and with a subarray of 100 or 128 rows to obtain time resolution of $0.3 \mathrm{~s}$ or $0.4 \mathrm{~s}$, respectively. XMM-Newton observations used the pn CCD in full-frame or large-window mode, with $74 \mathrm{~ms}$ or $44 \mathrm{~ms}$ resolution and, in most cases, the MOS CCDs in small-window mode with $0.3 \mathrm{~s}$ resolution. We corrected the processed archival XMM-Newton photons for leap seconds and time jumps when needed, and applied the barycentric correction at the VLBI measured position. We then computed frequencies and $1 \sigma$ errors using the $Z_{1}^{2}$ test on photons in the $0.3-4 \mathrm{keV}$ band. The two short Chandra HRC observations that were obtained for the purpose of source location have relatively uncertain frequencies, and were not used in the subsequent analysis.

The frequency measurements are shown in Figure 8(a). These were used to track the time-varying frequency derivative from 2003 to 2009 by computing the difference in frequency between 

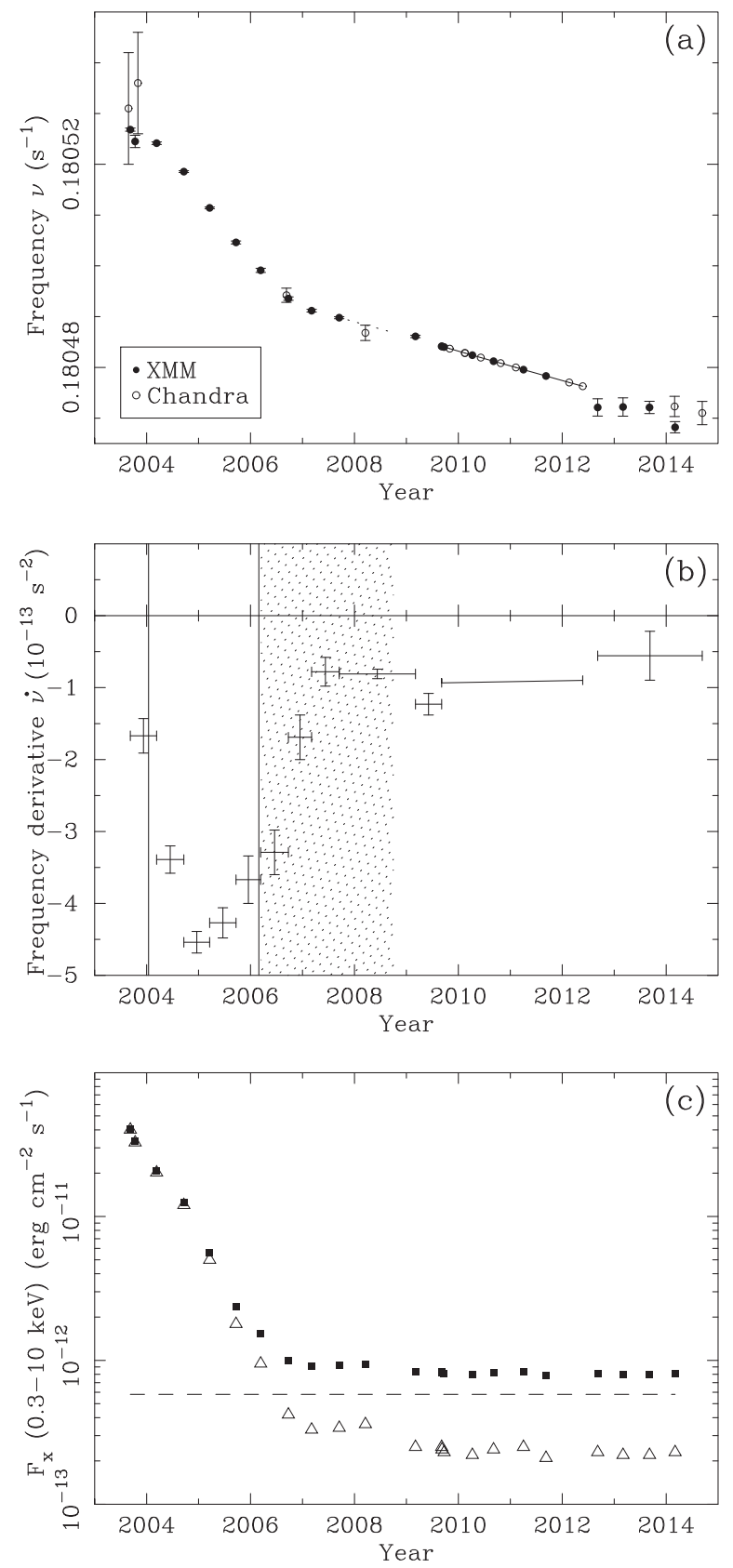

Figure 8. X-ray timing and flux properties of XTE J1810-197 from Chandra and XMM-Newton. (a) Frequency measurements, where the points linked with the solid line in 2009-2012 comprise the phase-connected solution in Table 5. The dashed line segment is the radio timing solution from Table 3. (b) Frequency derivatives obtained by differencing adjacent frequency measurements, and from the 2009-2012 phase-connected solution. The vertical lines denote the first two epochs of radio detection at $1.4 \mathrm{GHz}$ with the VLA (Halpern et al. 2005; Camilo et al. 2006), and the shaded region encompasses the epochs of pulsed radio detection from Figure 1. It is not known whether radio emission at the flux density level of the first detection $(4.5 \pm 0.5 \mathrm{mJy})$ was present earlier in the X-ray outburst, which was detected in 2003 January. (c) X-ray flux measurements from the three- or two-temperature blackbody fits of Alford \& Halpern (2016). Open triangles are the sum of the varying hot and warm areas, and filled squares are the total flux including the triangles and the cooler full surface area of the neutron star represented by the (constant) dashed line.

adjacent XMM-Newton observations, which were usually 6 months apart. Where these $\dot{\nu}$ measurements overlap with the more precise record obtained from radio observations during 2006-2008 (Figure 1(a)), they agree. Figure 8(b) illustrates that $\dot{\nu}$ varied by a factor of $\approx 6$. In the months immediately following
Table 5

X-Ray Timing Solution for XTE J1810-197

\begin{tabular}{ll}
\hline \hline Parameter & Value \\
\hline R.A. (J2000.0) & $18^{\mathrm{h}} 09^{\mathrm{m}} 51^{\mathrm{s}} .087$ \\
Decl. (J2000.0) & $-19^{\circ} 43^{\prime} 51^{\prime \prime} 93$ \\
Epoch (MJD TDB) & 55444.0 \\
Range of dates (MJD) & $55079-56071$ \\
Frequency, $\nu$ & $0.18048121539(63) \mathrm{Hz}$ \\
Frequency derivative, $\dot{\nu}$ & $-9.2121(35) \times 10^{-14} \mathrm{~Hz} \mathrm{~s}^{-1}$ \\
Frequency second derivative, $\ddot{\nu}$ & $4.1(3) \times 10^{-23} \mathrm{~Hz} \mathrm{~s}^{-2}$ \\
Surface dipole magnetic field, $B_{s}{ }^{\mathrm{a}}$ & $1.3 \times 10^{14} \mathrm{G}$ \\
Spin-down luminosity, $\dot{E}^{\mathrm{b}}$ & $6.6 \times 10^{32} \mathrm{erg} \mathrm{s}^{-1}$ \\
Characteristic age, $\tau_{c}^{\mathrm{c}}$ & $31 \mathrm{kyr}$ \\
\hline
\end{tabular}

Notes.

${ }^{\mathrm{a}} B_{s}=3.2 \times 10^{19}(P \dot{P})^{1 / 2} \mathrm{G}$, with $P$ in s, where $P=1 / \nu$.

${ }^{\mathrm{b}} \dot{E}=4 \pi^{2} \times 10^{45} \dot{P} / P^{3} \mathrm{erg} \mathrm{s}^{-1}$.

${ }^{\text {c }} \tau_{c}=P /(2 \dot{P})$.

the outburst, which was first detected in 2003 January, monitoring by $R X T E$ showed noisy spin-down with an even higher mean frequency derivative of $-6.7 \times 10^{-13} \mathrm{~Hz} \mathrm{~s}^{-1}$ (Ibrahim et al. 2004), which is $\approx 8$ times the minimum value of $\approx-8 \times 10^{-14} \mathrm{~Hz} \mathrm{~s}^{-1}$ measured by XMM-Newton in 2007-2008 (Figure 8(b)).

Starting in 2009 September, more frequent observations allowed a phase-connected solution to be established up through 2012 May. This process was facilitated by the relatively stable spin-down with a small rate at later times. Beginning with the two observations on 2009 September 5 and 7 , we folded the photons jointly using the $Z_{1}^{2}$ test and used the resulting frequency to fold the next observation, verifying that the predicted phase agreed with the observed one to $<0.2$ cycles. Each subsequent observation was then added to the joint fit and the $Z_{1}^{2}$ test was iterated with free parameters $\nu$ and $\dot{\nu}$, and finally $\ddot{\nu}$, until 15 observations were included. The resulting fit is shown by the solid line in Figure 8(a), and its $\dot{\nu}=-9.21 \times 10^{-14} \mathrm{~Hz} \mathrm{~s}^{-1}$ (Table 5) is very nearly a continuation of the minimum spin-down rate that was first reached in 2007. The second frequency derivative as listed in Table 5 is necessary to fit the 2009-2012 series with a continuous ephemeris.

Analyzing the same data from which we established the phase-connected ephemeris of Table 5, Pintore et al. (2016) claimed to identify a timing anomaly in which the pulsar was spinning up (positive $\dot{\nu}$ ) between 2010 September and 2011 February. This could have arisen from their underestimation of uncertainties in frequency measurements. In any case, our phase-coherent ephemeris spanning this time shows no such event. Pintore et al. (2016) also propose a single phaseconnected timing solution spanning all of 2007-2014. This is clearly invalid, because their listed uncertainties on the polynomial coefficients $(\nu, \dot{\nu}, \ddot{\nu})$ are two orders of magnitude larger than what would be needed to describe a unique cycle count. Also, the parameters of our actual phase-coherent timing segments in radio (Table 3 ) and X-ray (Table 5) disagree with theirs. In particular, their fitted $\dot{\nu}=-4.9 \times 10^{-14} \mathrm{~Hz} \mathrm{~s}^{-1}$ is about half the true value.

After 2012 May we were unable to maintain phase connection. The prior coherent timing solution fails to predict the phase of the 2012 September observation by $\approx 0.5$ cycles. It 


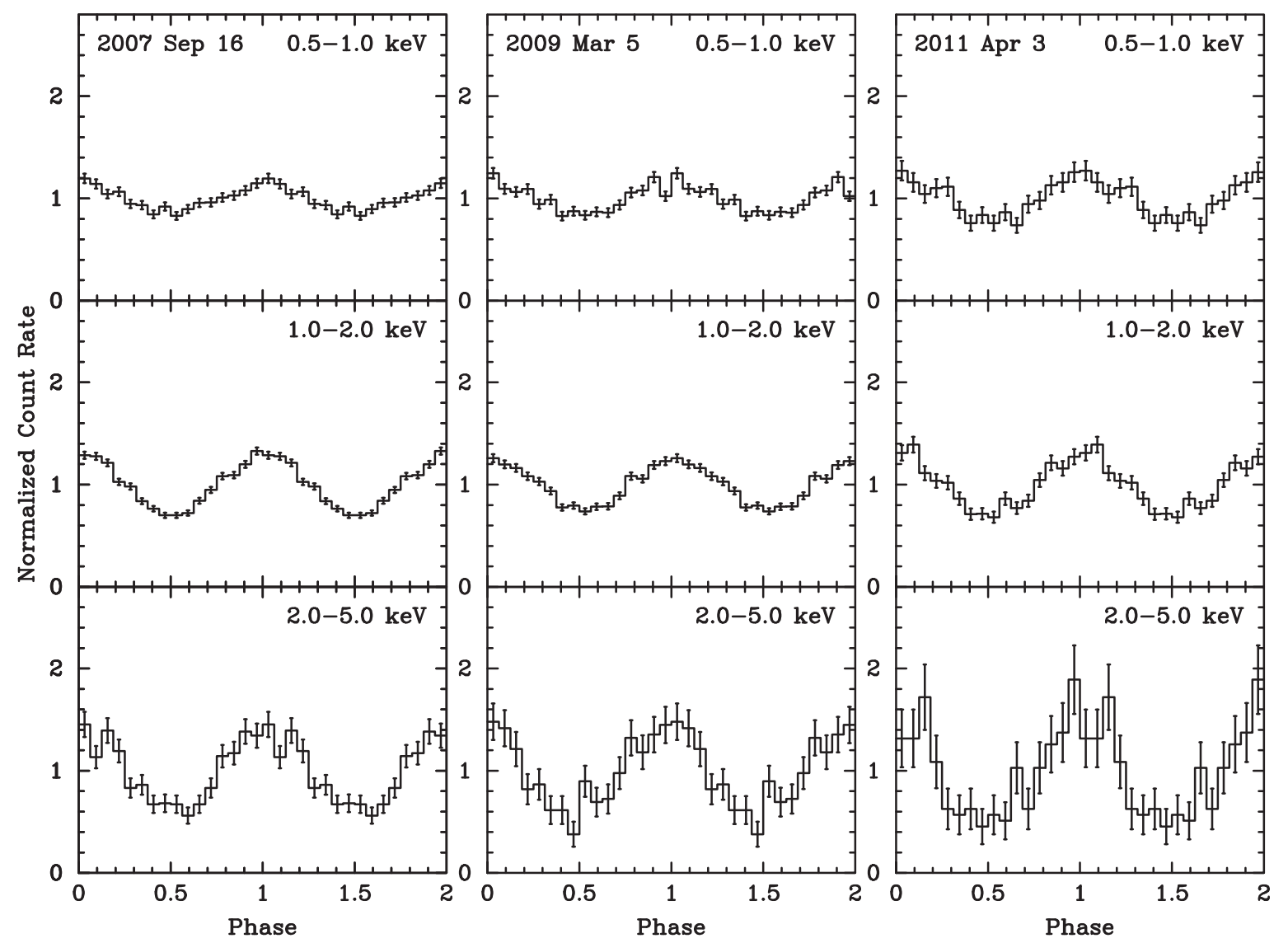

Figure 9. Energy-dependent pulse profiles of XTE J1810-197 from three XMM-Newton observations. Background has been subtracted, and the counts per bin are normalized so that the average is 1 in each panel. The profiles are centered on phase 1, which is arbitrary with respect to the radio phases displayed in Figures 6 and 7 .

is possible that an anti-glitch (Archibald et al. 2013; Şaşmaz Muş et al. 2014) and/or change in torque occurred between 2012 May and September. But we cannot tell for sure what happened because the change in frequency differs from the extrapolation by only $2 \sigma$. We can only estimate the frequency derivative from 2012 September to 2014 September with an incoherent fit to the frequencies, which is highly uncertain. All we can say is that the long-term frequency derivative did not clearly change in 2012 (see Figure 8(b)).

From 2009 September to 2012 May, $i$ changed by at most $4 \%$, as measured by the frequency second derivative (which is a factor of 6 smaller than that measured over the year following 2007 September; Table 3). Such behavior is common in AXPs and soft gamma repeaters (SGRs), which often show extended periods of smooth spin-down at small $|\dot{\nu}|$ and noisier epochs with larger $|\dot{\nu}|$ (Kaspi et al. 1999, 2001; Gavriil \& Kaspi 2002; Woods et al. 2002, 2007; Tam et al. 2008; Dib et al. 2009; Dib $\&$ Kaspi 2014). At one time, it was proposed that the combined action of free and radiative precession could explain the "bumpy" spin-down of AXPs (Melatos 1999, 2000), which predicted that $\dot{\nu}$ would oscillate with a period of several years. However, as observations of dramatic changes in $\dot{\nu}$ from magnetars have accumulated over the years, none appear to be dominated by such periodic variations (see references above). There is at best some evidence possibly indicating quasiperiodic $\dot{\nu}$ behavior in one magnetar (Archibald et al. 2015). The timing behavior of XTE J1810-197 shown in Figure 8 seems typical when compared with other magnetars that have been monitored for longer times with no cyclic pattern evident in their spin down.

Figure 9 shows three sample energy-dependent X-ray pulse profiles from XMM-Newton during (2007) and after (2009, 2011) the epoch of pulsed radio detection. They share the same characteristics as earlier observations, in particular those from 2005 and 2006 as shown in Gotthelf \& Halpern (2007). The pulse peaks are in phase as a function of energy, while the pulsed fraction increases sharply with energy. This is understood in a model in which a small hot spot is surrounded by a cooler, larger annulus that covers most of the neutron star. In summary, there is no obvious change in X-ray timing or pulse shapes corresponding to the shutdown of radio emission in late 2008.

\subsection{X-Ray Fluxes}

Figure $8(\mathrm{c})$ summarizes the results from blackbody spectral modeling that is described in more detail in Alford \& Halpern (2016). The spectrum is fitted with either two or three blackbodies, where the coolest is restricted to having a constant temperature and area representing the full surface of the neutron star (dashed line in Figure 8(c)). The triangles represent the fluxes from one or two blackbodies (hot and warm) with a much smaller area that account for the decaying outburst flux. The squares are the total flux at each epoch. It is evident that the fluxes have been steady since after 2009, following a slight decrease of $\approx 20 \%$ in the hot/warm component between 2008 March and 2009 March. This small step-down in flux, corresponding to $\sim 8 \times 10^{32} \mathrm{erg} \mathrm{s}^{-1}$ in bolometric luminosity, 
is the only event we have been able to find in X-rays that is approximately coincident with the turn-off of radio pulsations in late 2008.

\subsection{X-Ray and Radio Pulse Phase Alignment}

Five of the X-ray observations listed in Table 4 were made during the period in which XTE J1810-197 was an active radio source (see also Figure 8). Thus, for these observations we can compare the emission phases of the X-ray and radio profiles. For each of these observations (two made with Chandra and three with XMM-Newton), we used TEMPO to fit for an offset between the X-ray TOA and a small number of surrounding radio TOAs (the latter corrected to infinite frequency using the DM from Table 3). The radio reference phase is the peak of the main radio component, which, owing to changing pulse shapes, can contribute up to $0.02 P$ jitter in this comparison. The X-ray reference phase is the peak of the approximately sinusoidal profile, which has an effective uncertainty of up to $0.1 P$ for the Chandra observations and is a little better for the XMM-Newton profiles.

The measured offset between the X-ray and the radio TOAs was, in chronological order, $0.21,0.03,-0.17,0.01$, and $-0.28 \mathrm{~s}$. All radio and X-ray TOAs therefore match to within 0.05 P. Thus, at least between 2006 September and 2008 March, the peak of radio emission coincided with the peak of $\mathrm{X}$-ray emission.

\section{DISCUSSION}

\subsection{Geometry of the Rotating Neutron Star}

Since the main radio pulse coincides with the peak of the $\mathrm{X}$-ray emission, it is reasonable to assume that both radio and $\mathrm{X}$-ray are coming from near the surface, where a hot spot is located at the footpoint of a bundle of magnetic field lines (for the other two magnetars with detected radio and X-ray pulsations, the radio profile is not as well aligned with, but still overlaps, the X-ray profile; Halpern et al. 2008; Pennucci et al. 2015). In this picture, currents flowing along this field-line bundle are responsible for both surface heating and radio emission. The $\mathrm{X}$-ray and radio pulses should coincide as long as the field lines are normal to the surface and the radio emission height is much smaller than the radius of the speed of light cylinder. While these are reasonable assumptions for the open field-line bundle in ordinary pulsars, the geometry may be different in the case of magnetars, where currents on closed, twisted magnetic field lines are thought to contribute to X-ray emission via resonant cyclotron scattering (Lyutikov \& Gavriil 2006; Fernández \& Thompson 2007), and the location of the radio emission region is not obvious. Therefore, the rotating vector model (RVM; Radhakrishnan \& Cooke 1969) is not necessarily applicable unless the radio emission is produced on open field lines as in ordinary pulsars. Theory and observation are inconclusive as to whether radio emission in magnetars is produced on open or closed magnetic field lines (Thompson 2008).

Assuming that the RVM applies to magnetars, Camilo et al. (2007d) used it to deduce the geometry of the star. There were two possible solutions, depending on whether an orthogonal jump in polarization was introduced between widely spaced pulse components (P1 and P3 in Figure 6(a)). In the first solution, which did not include an orthogonal jump, the magnetic and rotational axes are almost aligned: $\alpha \approx 4^{\circ}$ and $\beta \approx 4^{\circ}$, where $\alpha$ is the angle between the magnetic and rotation axes, and $\beta$ is the angle of closest approach of the line of sight to the magnetic axis. The second solution, which inserted an orthogonal jump, yielded $\alpha \approx 70^{\circ}$ and $\beta \approx 25^{\circ}$.

New data reported here show a polarized component that was not present earlier (P2 in Figures 6(b) and (d), at a pulse phase between those of P1 and P3). If we combine the information on the PA values from different epochs (namely from data represented in Figures 6(a), (b), and (d)), we obtain RVM fits that are consistent with those presented in Camilo et al. (2007d). The result is again $\alpha \approx 4^{\circ}, \beta \approx 4^{\circ}$ with no orthogonal jump. The addition of an orthogonal jump between components P2 and P3 (see Figure 6(b)) yields $\alpha \approx 67^{\circ} \pm 10^{\circ}$ and $\beta \approx 16^{\circ} \pm 5^{\circ}$. Broadly speaking, therefore, the same arguments apply as before-either the pulsar is aligned, which is problematic given the X-ray pulse properties discussed below, or $\alpha$ is large and the emission height, determined from the width of the pulse, is also relatively large, $\sim 2 \times 10^{4} \mathrm{~km}$, which is about $8 \%$ of the light cylinder radius (see Camilo et al. 2007d).

It appears that the X-ray spectrum of XTE J1810-197 has always been dominated by the thermal hot spot and surrounding warm region (Gotthelf \& Halpern 2005; Halpern \& Gotthelf 2005), which is supported by the single-peaked $\mathrm{X}$-ray pulse that aligns well in phase as a function of energy. So the X-ray pulse has been modeled independently of the radio as an indicator of the spin orientation and viewing geometry of a surface hot spot, which can then be compared with the results of the RVM fits under the assumption that the hot spot underlies a perpendicular radio beam. The most recent such X-ray modeling results (Bernardini et al. 2011) allow, in our notation, $\alpha$ in the range $29^{\circ}-52^{\circ}$, which is degenerate with $\zeta=|\beta+\alpha|$, while $\beta$ ranges from $0^{\circ}$ to $23^{\circ}$. Their extreme solutions have $(\alpha, \zeta)=\left(29^{\circ}, 52^{\circ}\right)$ or $(\alpha, \zeta)=\left(52^{\circ}, 29^{\circ}\right)$. Neither of the RVM solutions are entirely consistent with the model of the X-ray pulse, although the high pulsed fraction of the harder X-rays would at least seem to rule out a nearly aligned rotator with $\alpha \sim 4^{\circ}$, and a geometry with ( $\alpha$, $\beta) \approx\left(52^{\circ}, 16^{\circ}\right)$ seems reasonably compatible with both radio and X-ray observations.

\subsection{Decline of the X-Ray and Radio Luminosity}

A detailed explanation for the exponential X-ray decay of XTE J1810-197 was developed by Beloborodov (2009). In this model, a bundle of closed, twisted magnetic field lines centered on the magnetic dipole axis carries the current that heats a spot on the surface. This so-called " $\mathrm{j}$-bundle" is the result of a twist of the crust by the starquake that initiated the outburst. As the j-bundle untwists, its boundary recedes toward the magnetic pole; thus, the area of its footpoint decreases, which accounts for the declining blackbody area fitted to the X-ray spectrum (Gotthelf \& Halpern 2007; Bernardini et al. 2009).

Beloborodov (2009) also accounts for the non-monotonic change in spin-down rate of XTE J1810-197, which after the outburst first increased then decreased as shown in Figure 8(b). The initial increase in torque is caused by a growing twist of field lines near the magnetic axis, even as the outer boundary of the $\mathrm{j}$-bundle is shrinking. This twist inflates the poloidal field lines, effectively increasing the dipole moment and the magnetic field strength at the light cylinder. Once the twist reaches a maximum stable value of $\sim 1$ radian, the dipole 
moment decreases due to the continued contracting of the $\mathrm{j}$-bundle, and the torque decreases.

In this picture, radio emission is produced on the closed field lines of the $\mathrm{j}$-bundle, which is wider and more energetic than the open field-line bundle. Therefore, radio emission from a transient magnetar in outburst could be easier to see, both for geometric and energetic reasons, than in its quiescent state. Its radio beam could be broader and different in many respects (spectrum, polarization, variability) from those of ordinary radio pulsars. The radio pulse in this model is coincident with the X-ray pulse, and when the j-bundle contracts to less than the width of the observed radio beam the radio emission should decrease rapidly.

However, in this model one may expect the width of the radio pulse to decrease gradually to zero as the $\mathrm{j}$-bundle shrinks, possibly approaching zero width and disappearing when the outer boundary of the emitting region reaches the tangent point to the observer's line of sight. But this is contrary to the observations, which show no change in the width or complexity of the radio pulse just before it disappeared. The X-ray flux from the hot/warm spot showed at most a $20 \%$ decrease $\left(\sim 8 \times 10^{32} \mathrm{erg} \mathrm{s}^{-1}\right)$ at the epoch of radio disappearance, when there was no detectable change in spin-down power in the X-ray timing. Because this luminosity change is comparable to or greater than the spin-down power at the time, it is difficult to understand how spin power could be responsible for the event. A more subtle physical process is probably required to explain the sudden quenching of the radio emission.

Rea et al. (2012) proposed that the (then) three radiodetected magnetars have a smaller quiescent X-ray luminosity than their spin-down power, but not the reverse: not all magnetars with $L_{x} / \dot{E}<1$ are radio pulsars. However, a review of these parameters for XTE J1810-197 shows that the minimum spin-down power of XTE J1810-197, which it reached in quiescence in 2007-2012, is in the range $\dot{E}=$ $(5.6-6.6) \times 10^{32} \mathrm{erg} \mathrm{s}^{-1}$; whereas its quiescent $(0.3-10 \mathrm{keV})$ $\mathrm{X}$-ray luminosity (uncorrected for absorption) is $L_{x} \approx 1 \times 10^{33} d_{3.5}^{2} \mathrm{erg} \mathrm{s}^{-1}$, both before and after the outburst (Gotthelf et al. 2004; Bernardini et al. 2011; Alford \& Halpern 2016). The distance of $3.5 \mathrm{kpc}$ is taken from Minter et al. (2008). The bolometric luminosity of the cool component alone from Alford \& Halpern (2016) is $4 \times 10^{34} d_{3.5}^{2} \mathrm{erg} \mathrm{s}^{-1}$. These values of $\dot{E}$ and $L_{x}$ disagree with those in Rea et al. (2012) and do not support their proposition, because the X-ray luminosity of XTE J1810-197 is greater than its spin-down power, whether before, during, or after the outburst.

Szary et al. (2015) explain radio pulsations from magnetars and ordinary radio pulsars by a single model: the partially screened gap. It assumes that rotational energy heats the openfield-line polar cap, and the resulting temperature, compared with a critical temperature for ion emission, is what determines whether a partially screened gap is maintained. Only if the luminosity of the polar cap is much less than the spin-down power is radio emission possible. However, the temperature of the X-ray/radio-emitting cap in XTE J1810-197 is larger than that of a rotation-powered pulsar with the same timing parameters because it is heated by magnetic field decay, not by rotation. During the outburst of XTE J1810-197 the polar cap luminosity rose by more than two orders of magnitude, while the spin-down luminosity only increased by a factor of 8 . So it is not clear how this model could explain the onset or turn-off of radio pulsations during the outburst of XTE J1810-197.

\section{CONCLUSIONS}

Radio pulsations appear to be characteristic of some, but not all transient magnetars in outburst. The long record left by the single known outburst of XTE J1810-197 provides a prototype for investigating the mechanism of magnetar radio emission. The radio flux densities and X-ray fluxes each declined by about a factor of 50 from the peak of the outburst to the year 2006. Then the X-ray and radio luminosities both leveled off in 2007. This large-amplitude correlation would argue that the power for the radio emission comes from the magnetar mechanism that creates currents in the pulsar magnetosphere and heats the neutron star crust, rather than from rotation power.

Before the radio pulses of XTE J1810-197 turned off, they continued to show large day-to-day fluctuations, unlike ordinary radio pulsars. The radio spectrum appears to have changed from flat to steep as the radio (and X-ray) emission leveled off. The emission remained highly polarized and the observation of a new polarized pulse component allowed us to test and refine the previously derived emission geometry, assuming a dipole field geometry. This was compared with independent modeling of the X-ray pulse, which is coincident in phase with the main radio pulse. The radio polarization allows two solutions, depending on whether an orthogonal jump in polarization is assumed between pulse components. However, the almost aligned solution appears inconsistent with the large-amplitude X-ray pulse, so we favor the more inclined model.

Finally, the radio pulsations turned off abruptly in late 2008, and have not reappeared in the subsequent 7 years. However, a continuing pulse of hard X-rays from a hot spot persists during the radio quiet epoch and exceeds the spin-down luminosity, which is evidence of continuing magnetar activity. This should not be thought of as a "return to quiescence," because magnetar activity is not a quiescent state, but as a continuing conversion of magnetic energy to luminosity that, for a period of years, may well result in a quasi-constant luminosity. We also do not know enough about the pre-outburst state of XTE J1810 -197 to determine whether it had precisely the same emission properties then as it does now, or if it was truly quiescent prior to the outburst detected in early 2003.

The sudden radio disappearance prompted us to search for any contemporaneous event in the X-ray record that could be associated with it. The only possible such occurrence was a step-down in the X-ray flux of the hot spot by $\approx 20 \%$ between 2008 March and 2009 March. Although this would appear to be a small effect, it bears some consideration, because none of the other observations and theories offer a natural explanation for the sharp radio turn-off.

The National Radio Astronomy Observatory is a facility of the National Science Foundation operated under cooperative agreement by Associated Universities, Inc.

The Nançay Radio Observatory is operated by the Paris Observatory, associated with the French Centre National de la Recherche Scientifique (CNRS).

The Parkes Observatory is part of the Australia Telescope, which is funded by the Commonwealth of Australia for operation as a National Facility managed by CSIRO. 
This investigation is partly based on observations obtained with $X M M-N e w t o n$, an ESA science mission with instruments and contributions directly funded by ESA Member States and NASA, and with Chandra. The Chandra X-ray Observatory Center is operated by the Smithsonian Astrophysical Observatory for and on behalf of NASA under contract NAS8-03060. This research also made use of data obtained from the High Energy Astrophysics Science Archive Research Center (HEASARC), provided by NASA's Goddard Space Flight Center. J.P.H. acknowledges support from NASA ADAP grant NNX15AE63G.

Facilities: GBT (Spigot, BCPM), NRT (BON), Parkes (DFB, AFB), CXO (ACIS-S), XMM (pn, MOS).

\section{REFERENCES}

Alford, J. A. J., \& Halpern, J. P. 2016, ApJ, 818, 122

Archibald, R. F., Kaspi, V. M., Ng, C.-Y., et al. 2013, Natur, 497, 591

Archibald, R. F., Kaspi, V. M., Ng, C.-Y., et al. 2015, ApJ, 800, 33

Baars, J. W. M., Genzel, R., Pauliny-Toth, I. I. K., \& Witzel, A. 1977, A\&A, 61, 99

Beloborodov, A. M. 2009, ApJ, 703, 1044

Bernardini, F., Israel, G. L., Dall'Osso, S., et al. 2009, A\&A, 498, 195

Bernardini, F., Perna, R., Gotthelf, E. V., et al. 2011, MNRAS, 418, 638

Camilo, F., Cognard, I., Ransom, S. M., et al. 2007a, ApJ, 663, 497

Camilo, F., Ransom, S. M., Halpern, J. P., \& Reynolds, J. 2007b, ApJL, 666, L93

Camilo, F., Ransom, S. M., Halpern, J. P., et al. 2006, Natur, 442, 892

Camilo, F., Ransom, S. M., Peñalver, J., et al. 2007c, ApJ, 669, 561

Camilo, F., Reynolds, J., Johnston, S., Halpern, J. P., \& Ransom, S. M. 2008, ApJ, 679, 681

Camilo, F., Reynolds, J., Johnston, S., et al. 2007d, ApJL, 659, L37

Cognard, I., \& Theureau, G. 2006, in 26th Meeting of the IAU 36, JD02, On the Present and Future of Pulsar Astronomy, 2

Dib, R., \& Kaspi, V. M. 2014, ApJ, 784, 37

Dib, R., Kaspi, V. M., \& Gavriil, F. P. 2009, ApJ, 702, 614

Duncan, R. C., \& Thompson, C. 1992, ApJL, 392, L9

Fernández, R., \& Thompson, C. 2007, ApJ, 660, 615

Gavriil, F. P., \& Kaspi, V. M. 2002, ApJ, 567, 1067

Gotthelf, E. V., \& Halpern, J. P. 2005, ApJ, 632, 1075

Gotthelf, E. V., \& Halpern, J. P. 2007, Ap\&SS, 308, 79
Gotthelf, E. V., Halpern, J. P., Buxton, M., \& Bailyn, C. 2004, ApJ, 605,368

Halpern, J. P., \& Gotthelf, E. V. 2005, ApJ, 618, 874

Halpern, J. P., Gotthelf, E. V., Becker, R. H., Helfand, D. J., \& White, R. L. 2005, ApJL, 632, L29

Halpern, J. P., Gotthelf, E. V., Reynolds, J., Ransom, S. M., \& Camilo, F. 2008, ApJ, 676, 1178

Helfand, D. J., Chatterjee, S., Brisken, W. F., et al. 2007, ApJ, 662, 1198

Hotan, A. W., van Straten, W., \& Manchester, R. N. 2004, PASA, 21, 302

Ibrahim, A. I., Markwardt, C. B., Swank, J. H., et al. 2004, ApJL, 609, L21

Kaplan, D. L., Escoffier, R. P., Lacasse, R. J., et al. 2005, PASP, 117, 643

Kaspi, V. M., Chakrabarty, D., \& Steinberger, J. 1999, ApJL, 525, L33

Kaspi, V. M., Gavriil, F. P., Chakrabarty, D., Lackey, J. R., \& Muno, M. P. 2001, ApJ, 558, 253

Keith, M. J., Johnston, S., Levin, L., \& Bailes, M. 2011, MNRAS, 416, 346

Kramer, M., Stappers, B. W., Jessner, A., Lyne, A. G., \& Jordan, C. A. 2007, MNRAS, 377, 107

Lazaridis, K., Jessner, A., Kramer, M., et al. 2008, MNRAS, 390, 839

Levin, L., Bailes, M., Bates, S., et al. 2010, ApJL, 721, L33

Lorimer, D. R., \& Kramer, M. 2004, Handbook of Pulsar Astronomy (Cambridge: CUP)

Lyutikov, M., \& Gavriil, F. P. 2006, MNRAS, 368, 690

Manchester, R. N., Hobbs, G., Bailes, M., et al. 2013, PASA, 30, e017

Manchester, R. N., Lyne, A. G., Camilo, F., et al. 2001, MNRAS, 328, 17

Melatos, A. 1999, ApJL, 519, L77

Melatos, A. 2000, MNRAS, 313, 217

Minter, A. H., Camilo, F., Ransom, S. M., Halpern, J. P., \& Zimmerman, N. 2008, ApJ, 676, 1189

Olausen, S. A., \& Kaspi, V. M. 2014, ApJS, 212, 6

Pennucci, T. T., Possenti, A., Esposito, P., et al. 2015, ApJ, 808, 81

Pintore, F., Bernardini, F., Mereghetti, S., et al. 2016, MNRAS, 458, 1190

Radhakrishnan, V., \& Cooke, D. J. 1969, ApL, 3, 225

Rea, N., Pons, J. A., Torres, D. F., \& Turolla, R. 2012, ApJL, 748, L12

Reich, P., Testori, J. C., \& Reich, W. 2001, A\&A, 376, 861

Şaşmaz Muş, S., Aydın, B., Göğüş, E., et al. 2014, MNRAS, 440, 2916

Serylak, M., Stappers, B. W., Weltevrede, P., et al. 2009, MNRAS, 394, 295

Shannon, R. M., \& Johnston, S. 2013, MNRAS, 435, L29

Szary, A., Melikidze, G. I., \& Gil, J. 2015, MNRAS, 447, 2295

Tam, C. R., Gavriil, F. P., Dib, R., et al. 2008, ApJ, 677, 503

Theureau, G., Coudreau, N., Hallet, N., et al. 2005, A\&A, 430, 373

Thompson, C. 2008, ApJ, 688, 499

Woods, P. M., Kouveliotou, C., Finger, M. H., et al. 2007, ApJ, 654, 470

Woods, P. M., Kouveliotou, C., Göğüş, E., et al. 2002, ApJ, 576, 381 